\title{
Maturation of the infant rhesus macaque gut microbiome and its role in the development of diarrheal disease
}

\author{
Nicholas Rhoades ${ }^{1}$, Tasha Barr ${ }^{1}$, Sara Hendrickson² ${ }^{2}$ Kamm Prongay ${ }^{3}$, Andrew Haertel ${ }^{3}$, Leanne Gill ${ }^{4}$, Laura Garzel ${ }^{4}$, \\ Katrine Whiteson', Mark Slifka² and Ilhem Messaoudi ${ }^{*}$
}

\begin{abstract}
Background: Diarrhea is the second leading cause of death in children under 5 years of age. Enhanced understanding of causal pathways, pathogenesis, and sequelae of diarrhea is urgently needed. Although the gut microbiota is believed to play a role in susceptibility to diarrheal diseases, our understanding of this association remains incomplete. Infant rhesus macaques (Macaca mulatta) are susceptible to diarrhea making them an ideal model to address this question.

Results: The maturation of the infant rhesus macaque gut microbiome throughout the first 8 months of life occurs in a similar pattern as that described for human infants. Moreover, the microbiome of the captive reared infant rhesus macaque more closely resembles that of human infants in the developing world than in the western world. Importantly, prior to disease onset, the gut microbiome of infants that later develop diarrhea is enriched in pathways of immunomodulatory metabolite synthesis, while those of infants that remain asymptomatic are enriched in pathways for short-chain fatty acid production. We identify Prevotella strains that are more abundant at 1 month in infants that later develop diarrhea. At 8 months, the microbiomes of animals that experience diarrhea show increased abundance of Campylobacter and a reduction in Helicobacter macacae.
\end{abstract}

Conclusion: The composition of the microbial community could provide a phenotypic marker of an infant's susceptibility to diarrheal disease. Given the significant physiological and immunological similarities between human and nonhuman primates, these findings provide potential markers of susceptibility to diarrhea that could be modulated to improve infant health, especially in the developing world.

\section{Introduction}

The human body is a host to a diverse microbial community collectively known as the gut microbiota that is composed of trillions of microbial cells. These microbes encode far more genetic diversity than the human genome and play an essential role in host physiology [1-3]. The gut microbial community ferments indigestible substrates yielding energy and vitamins previously inaccessible to the host $[4,5]$. Commensal microbes also communicate with the host immune system, outcompete pathogens, and produce small molecules that modulate physiological functions locally or systemically. For example, butyrate is utilized locally in the gut, while

\footnotetext{
* Correspondence: imessaou@uci.edu

${ }^{1}$ Department of Molecular Biology and Biochemistry, University of California Irvine, Irvine, CA, USA

Full list of author information is available at the end of the article
}

tryptophan is converted to serotonin by gut enterochromaffin cells and acts systemically [6-9]. The gut microbial community is shaped by many host genetic and environmental factors such as diet, antibiotic use, social interactions, and sanitation practices. A prime example of this is humans living a modern western lifestyle have a distinct and less-diverse gut microbial community compared to individuals living in the developing world [10-12]. This variation emphasizes the difficulty in defining what a normal healthy community is [13, 14]. While the gut microbiome has been implicated in multiple chronic, acute, and infectious diseases [15-17], its potential as a therapeutic or biomarker for disease susceptibility is difficult to determine in humans.

The human gut is initially colonized at birth through exposure to microbes from humans and the environment. The identity of the initial colonizers may be 
affected by the mode of delivery [18, 19], as well as breastfeeding which lead to dominance by Bifidobacteria that can break down human milk oligosaccharides (HMOs) [20-22]. Additionally, Bifidobacteria internalize nutrients such as HMOs before degrading them, thereby sequestering the nutrients and decreasing the potential for enteropathogens to cross-feed on intermediate breakdown products [23]. Disruptions in this process due to, for instance, the early use of antibiotics have been implicated in the development of metabolic and autoimmune disorders [24-26]. Reduced microbial exposure in early infancy may contribute to the observed increase in allergies in the developed world [27, 28].

Diarrheal diseases cause significant morbidity and mortality in young children and result in malabsorption of nutrients [29], loss of barrier function [30], growth stunting [31], impaired brain development [32], and poor response to oral vaccines [33]. A wide range of enteropathogens (Rotavirus, Norovirus, Campylobacter, etc.) are responsible for diarrheal diseases in children under 5 years of age. However, these pathogens only account for $40-50 \%$ of the cases leaving a substantial number that cannot be definitely attributed to a specific pathogen [34-37]. The composition of early microbial community could represent a phenotypic marker for an individual's susceptibility to diarrheal diseases and response to treatment. Indeed, perturbations of this community early in life can be detrimental $[24,25,38-42]$. A model system that faithfully recapitulates the hallmarks of infant diarrheal diseases would provide a better understanding of other susceptibility factors and is needed to design interventions and treatments.

The gut microbiomes of captive nonhuman primates (NHPs) show similarities to those of humans in developing countries [43, 44]. Specifically, the adult rhesus macaque (Macaca mulatta) gut microbiome is enriched in the genera Prevotella, Ruminococcus, and Treponema, while almost completely lacking the genus Bacteroides largely found in westernized humans [10]. Importantly, captive outdoor-housed rhesus macaque infants experience a spectrum of acute and recurrent diarrheal disease that mimics enteric diseases found among children living in the developing world [45]. Infant rhesus macaque experience higher rates of diarrhea and more severe disease compared to adults [45]. Furthermore, NHPs including rhesus macaques have greater quantities and diversity of milk oligosaccharides that promote the growth of specific Bifidobacteria in the infant gut compared to humans [46, 47]. Studies on the infant rhesus microbiome have been limited, reporting a decrease in Epsilonproteobacteria associated with maternal high-fat diet during gestation [48], fluctuations in the abundance of Prevotella based on social interactions [49], and an increase in Th17 cells in the peripheral blood of breast-fed compared to formula-fed infant macaques [50]. However, none of these studies investigated diarrheal disease.

Here, we characterize the maturation of the infant rhesus macaque gut microbiome over the first 8 months of life in a large group of captive outdoor-housed animals at the Oregon and California National Primate Research Centers (ONPRC and CNPRC respectively). Our data suggest that the gut microbiome of outdoor-housed infant rhesus macaques is similar to that of humans living in the developing world thereby providing a suitable model for the study of diarrheal diseases, which disproportionally affect these countries. We compared the gut microbiome of animals that developed diarrhea to those that did not at two time points: (1) 1 month of age (before the onset of disease) and (2) 8 months (after disease incidence and treatment). We report that at 1 month of age the microbial community in infants that later developed diarrhea were functionally different and harbored unique Prevotella strains compared to that of infants that remained asymptomatic. At 8 months of age, animals that experienced clinical diarrhea harbored a larger burden of Campylobacter despite case-specific treatment with antibiotics and probiotics. Both direct culture and shotgun metagenomics revealed the presence of several Campylobacter species in fecal samples obtained only from animals that experienced diarrhea. These data illustrate how the rhesus macaque model can enhance our understanding of diarrheal disease pathogenesis, and support improved diagnostics and treatments.

\section{Results}

Infant rhesus macaques harbor distinct gut microbiome based on host age

We characterized the maturation of the rhesus macaque gut microbiota by high-throughput amplicon sequencing of the 16S rRNA gene (V4 region) of 313 rectal swabs collected from 40 dam/infant pairs housed at the ONPRC and CNPRC (Fig. 1a). To test for differences in overall microbial community composition, we utilized both unweighted (binary) and weighted (abundance based) UniFrac, a taxonomically derived measurement of similarity between microbial communities. Dissimilarity matrices of both unweighted and weighted UniFrac metrics were created and plotted using principal coordinate analysis (PcoA) (Fig. 1b and Additional file 1: Figure S1A, respectively). Since we collected samples from animals at two different sites, we determined the contribution of age and location to the variation within UniFrac dissimilarity matrices using a PERMANOVA. This analysis revealed that age explained a much more significant amount of total variation (7.6-10.2\%) compared to location (0.7$1.3 \%$ ) or individual animal $(0.5-0.7 \%)$ (Fig. 1c). This 


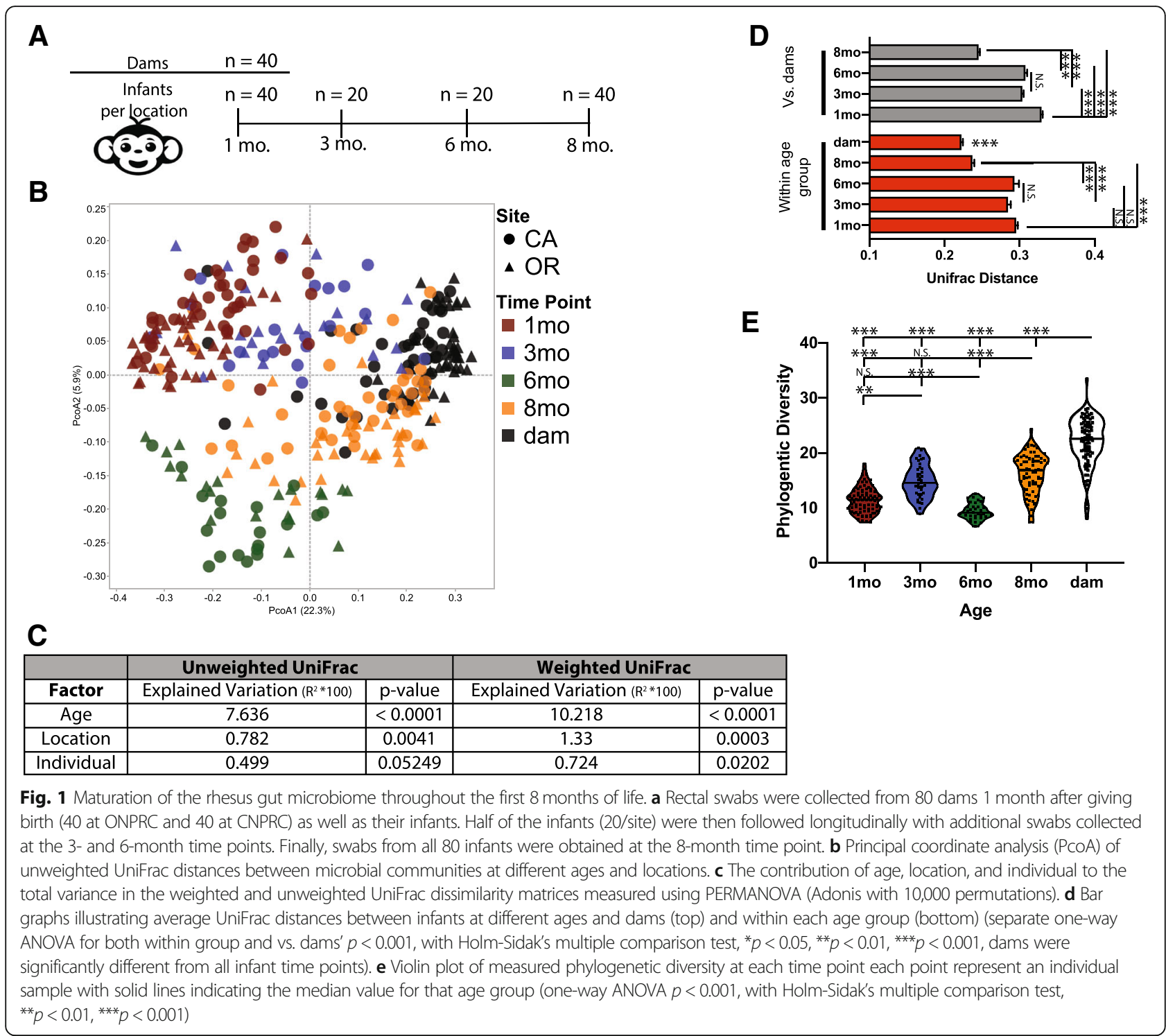

indicates that the microbiome of outdoor-housed captive macaques raised in a Northwest climate (Oregon) is similar to the microbiome of animals raised in a more arid Southwest climate (California).

Since age was the dominant factor driving variation, samples from both ONPRC and CNPRC were merged to increase power for detecting age-related trends. Over the course of 8 months, the microbiomes of infants became more similar to those of their dams (Fig. 1b, d). As reported in humans $[10,51]$, younger infants (1-3 months) showed larger intergroup differences than older infants (6-8 months) when compared to dams (Fig. 1b, d). Moreover, all infants showed more intragroup variation than the dams, but this variation decreased with age (Fig. 1d). Next, we used multiple alpha diversity metrics to assess changes in composition of this microbial community. We found that phylogenetic diversity increased with age, with the exception of a decrease in diversity at the 6-month time point (Fig. 1e) and similar patterns were observed in observed OTUs and Shannon evenness (Additional file 1: Figure S1B and C). The microbiomes of the dams exhibited higher phylogenetic diversity than infants at all time points, suggesting that the gut microbiome of infant macaque continues to develop beyond 8 months of age (Fig. 1e).

The infant macaque gut microbiome is more similar to that of children living in the developing world than children living in developed countries

We next compared the gut microbiome of infant rhesus macaques to those of human infants and children living in developing and developed countries using previously 
published datasets [10,11]. First, we compared the gut microbiome of pre-weaned 1-month-old macaques to that of human infants 6 months to 2 years of age from Malawi, Amerindians from Venezuela, and the USA (Fig. 2a). This analysis indicated that the gut microbiome of young infant macaques was more similar to that of human infants in developing countries (Fig. 2b). Second, we compared the gut microbiome of weaned 8-monthold infant macaques to that of children 2-6 years old from developing (Malawi, Burkina Faso, and the Amerindians from Venezuela) and developed countries (USA and Italy) (Fig. 2c). As described for 1-month-old samples, the gut microbiome of 8-month-old infant macaques was more similar to that of children living in developing countries than western countries (Fig. 2d). Interestingly, the gut microbiome of human children in developing countries was more similar to that of 8month-old macaques than to that of children living in developed countries (Fig. 2d).
The taxonomic landscape of the rhesus macaque gut microbiome

We next defined the taxonomic landscape of the rhesus macaque gut microbiome at different ages to identify the taxa driving differences in overall diversity over time. At the phyla level, regardless of age, the rhesus macaque microbiota was dominated by Bacteroidetes (primary genus Prevotella) and Firmicutes (Fig. 3a). The high prevalence of Prevotella in the microbiome of the captive macaque is likely due to the low percentage of animal fats in the monkey chow given the susceptibility of this species to bile acids secreted in response to meat consumption [52]. In contrast, Actinobacteria (primary genus Bifidobacteria) and Spirochetes (primary genus Treponema) displayed opposing age-dependent trends (Fig. 3b). Bifidobacterium plays a key role in the metabolism of breast milk oligosaccharides [53] and their disappearance at the 6-month time point coincides with when most infant macaques are weaned. Inversely, the

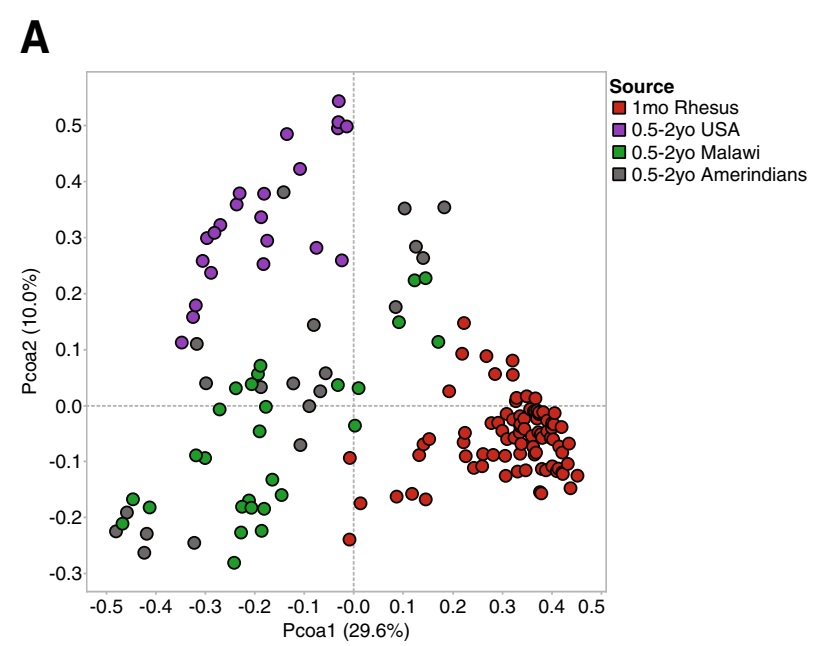

C

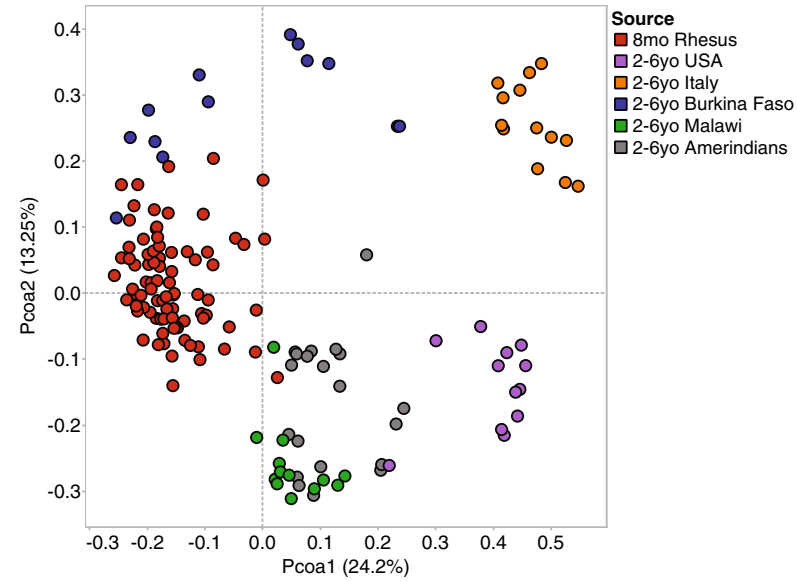

B

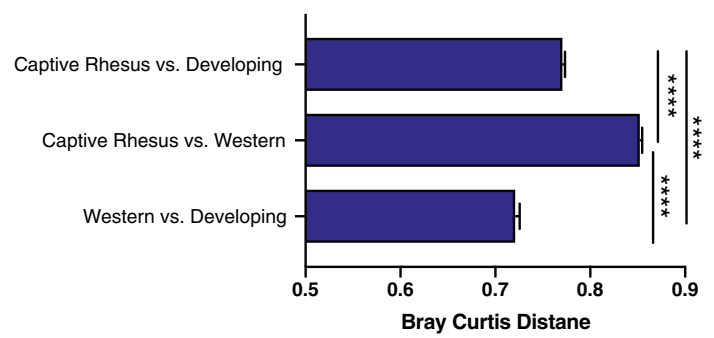

D

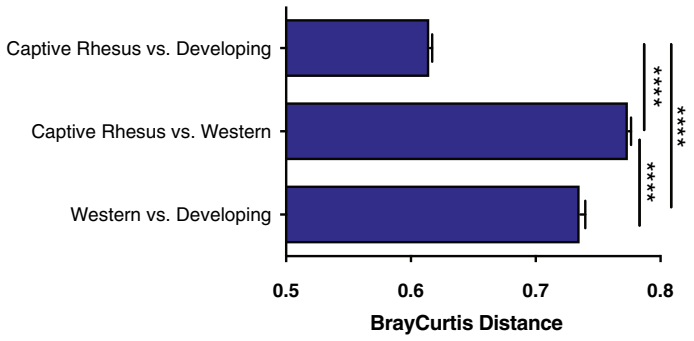

Fig. 2 Similarity of the infant macaque gut microbiome to human children. a Principal coordinate analysis (PcoA) of Bray-Curtis distances between gut microbial communities of pre-weaned 1-month-old infant rhesus macaque and human infants between 6 months and 2 years of age from the USA (western), Malawi (developing), and Amerindians (developing) at the genus (L6) level. b Bar graphs illustrating the average Bray-Curtis distances between 1month-old infant macaques and human (6 months-2 years) from western (USA) and developing (Malawi, Amerindians) countries (one-way ANOVA $p<$ 0.001 , with Holm-Sidak's multiple comparison test, ${ }^{* * *} p<0.0001$, error bars $\left.=S E M\right)$. $\mathbf{c}$ Principal coordinate analysis $(P c O A)$ of Bray-Curtis distances between gut microbial communities of post-weaned 8-month-old infant rhesus macaque and human infants between 2 and 6 years of age from the USA (western), Italy (western), Malawi (developing), Amerindians (developing), and Burkina Faso (developing) at the genus (L6) level. d Bar graphs illustrating the average Bray-Curtis distances between 8-month-old infant macaques and human (2-6years old) from western (USA and Italy) and developing (Malawi, Amerindians, and Burkina Faso) countries (one-way ANOVA $p<0.001$, with Holm-Sidak's multiple comparison test, ${ }^{* * *} p<0.0001$, error bars $=$ SEM) 


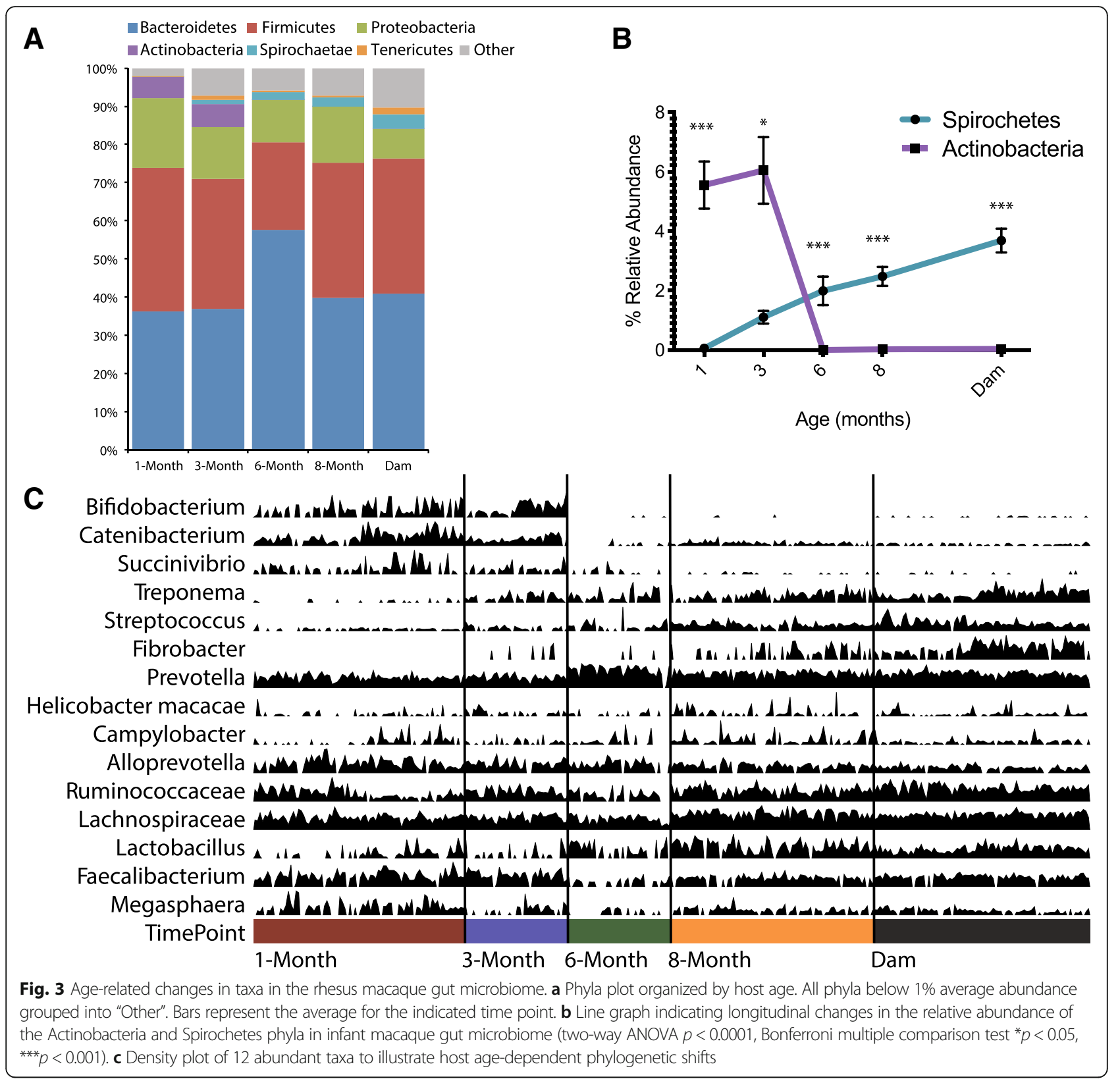

relative abundance of Spirochetes increased steadily throughout the 8 months (Fig. $3 \mathrm{~b}$ ). We explored finerscale taxonomy by pairwise age-group comparisons using LEfSe [54]. Comparing the microbiomes of all infants (1-8 months) to those of the dams identified 132 significantly different taxa (Additional file 2: Table S1). Of importance, the genera Fibrobacter, Treponema, and Lactobacillus were enriched in dams, most likely due to the consumption of a high-fiber chow [55]. On the other hand, Campylobacter, Bifidobacterium, Catenibacterium, Succinivibrio, and Helicobacter were more abundant in infants (Fig. 3c and Additional file 2: Table S1).
Diarrhea and antibiotic treatment results in a dysbiotic gut microbiome

Over the course of the study, $18.75 \%$ (15/80) of infants were hospitalized with clinical diarrhea and required veterinary care including oral hydration, antibiotics, and probiotics administered on a case-by-case basis (Additional file 2: Table S2). Approximately 47\% (7/15) of the diarrhea cases involved 2 or more episodes resulting in repeated hospitalization. Approximately $70 \%$ of the cases were initially diagnosed as Campylobacter coli-associated diarrhea (two cases were diagnosed with both $C$. coli and Shigella flexneri), 9\% were diagnosed with Campylobacter lari-associated diarrhea, 
4\% were diagnosed with $S$. flexneri alone (one case), and $17 \%$ had no definitive diagnosis at the time of their first hospitalization.

As recently reported in an independent cohort [56], diarrhea was associated with physical growth faltering as these 15 infants weighed significantly less than infants that remained asymptomatic at 6 and 8 months of age (Fig. 4a). We compared the microbiomes (defined using $16 \mathrm{~S}$ rRNA gene amplicon sequencing) of these 15 infants to those of asymptomatic infants that never developed clinical diarrhea at the 1-month time point (pre-diarrhea, $n=15$ diarrhea and 65 asymptomatic) to elucidate potential susceptibility biomarkers and at the 8-month time point (post-diarrhea, $n=12$ diarrhea and 62 asymptomatic) to determine the impact of diarrhea and associated treatments. At the 1-month time point (pre-diarrhea), we detected no compositional differences between the microbiomes of these two groups using any of the alpha/beta diversity metrics (Fig. 4b, c, Additional file 1: Figure S1D). Two taxa were differentially abundant at the 1-month time point: Lactobacillus salivarius and Haemophilius spp. were enriched in infants that remained asymptomatic and those that later experienced at least one diarrhea episode respectively (Additional file 2: Table S3).

In contrast, at the 8-month time point, the microbiomes of infants that experienced at least one episode

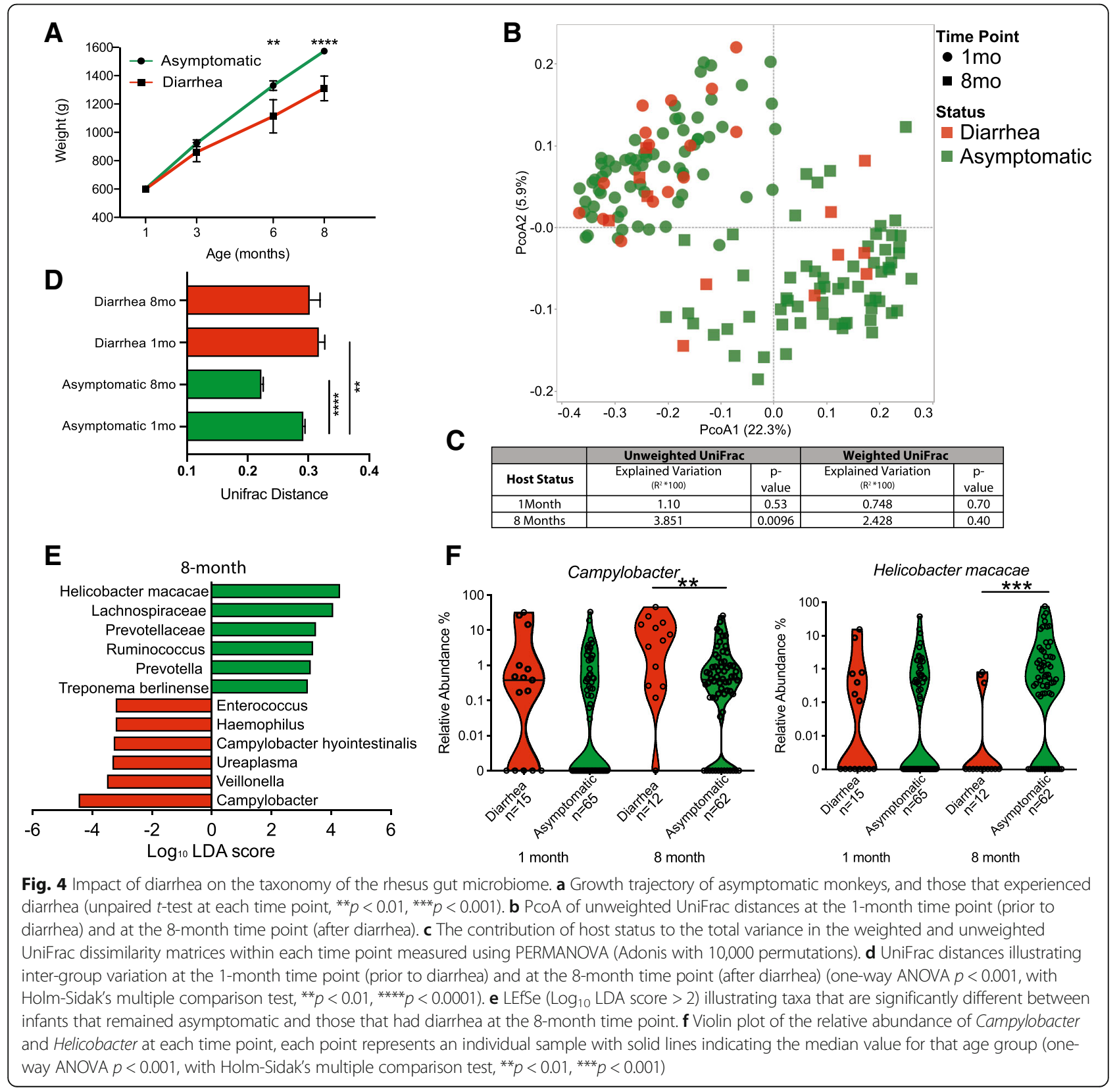


of diarrhea and associated treatment were distinct from those of asymptomatic infants that did not develop diarrhea based on unweighted UniFrac dissimilarity but not weighted Unifrac or Phylogenetic diversity (Fig. 4b, c, Additional file 1: Figure S1D). Additionally, the intragroup variation between the 1- and 8-month time points as measured by 1-way ANOVA using HolmSidak's multiple comparison test of Unifrac distance decreased within the asymptomatic group but not in the infant that experienced diarrhea (Fig. 4d). Moreover, at the 1-month time point, infants that would go on to experience diarrhea had higher intragroup variability than asymptomatic infants at the same time point (Fig. 4d). Additionally, at the 8-month time point, 30 taxa were differentially abundant between infants that remained asymptomatic and those that experienced at least one episode of diarrhea (Fig. 4e, Additional file 2: Table S3). Notably, the microbiomes of infants that experienced diarrhea were enriched for the genus Campylobacter, while those of asymptomatic animals were enriched in Helicobacter macacae (Fig. 4f). This is consistent with the detection of Campylobacter in the majority of the diarrhea in cases (Additional file 2: Table S2). Additional analysis showed that both of these genera were detected in 307 of 320 samples and at a relative abundance of greater than $2 \%$ in 104 samples. Interestingly, these two genera only co-occurred at a relative abundance of greater than $2 \%$ in only 3 out of 104 samples, suggestive of potential niche competition (Additional file 1: Figure S1E and F).

\section{Shotgun metagenomics reveal taxonomic and functional biomarkers of diarrhea susceptibility and differences induced by diarrhea and antibiotic treatment}

Shotgun sequencing provides higher strain level resolution than $16 \mathrm{~S}$ amplicon sequencing and elucidates functional potential of the total gut microbial community. Therefore, to better assess the microbial shifts associated with diarrhea/treatment, we next used shotgun metagenomics to compare the microbiomes of the infants that developed diarrhea and a subset of those that remained asymptomatic both at the 1-month time point (prior to the onset of disease, 6 asymptomatic and 5 diarrhea) and at the 8-month time point (after disease resolution, 10 asymptomatic and 7 diarrhea). While the overall taxonomic composition was not found to be significantly different based on host status at either time point (Additional file 1: Figure S2A and B). Several bacterial species were differentially abundant between these two groups at both time points. At the 1month time point, Mitsuokella spp. and Lachnospiraceae were more abundant in infants that remained asymptomatic, while abundance of Roseburia intestinalis was higher in those that later developed diarrhea (Additional file 1: Figure S2C). At the 8-month time points, the microbiomes of infants that remained asymptomatic were enriched for Helicobacter macacae, Lactobacilli johnsonii, Ruminococcus callidus, and Treponema succinifaciens species and other commensals (Additional file 1: Figure S2C). On the other hand, microbiomes of 8-month-old animals that experienced diarrhea were enriched in Acidaminococcus intestni, a bacterium associated with growth faltering in human children [57] and Lachnospiraceae (5163FAA) (Additional file 1: Figure S2D). In contrast to the culture-based results (Additional file 2: Table S2) and 16S data (Fig. 4e), this analysis did not identify Campylobacter as a differentially abundant genus. This is most likely due to the lack of rhesus macaque-specific Campylobacter genomes in the MetaPhlan2 database.

Despite minimal taxonomic differences, the microbiomes of infants that later developed diarrhea were functionally distinct from those that remained asymptomatic (Fig. 5a, b). At the 1-month time point, 63 pathways were differentially abundant between animals that remained asymptomatic and those that later developed diarrhea (Fig. 5c and Additional file 2: Table S4). Specifically, the microbiomes of animals that remained asymptomatic were enriched in pyruvate fermentation pathways important for the production of short chain fatty acid (SCFA), and pathways specific to Bifidobacterium. In contrast, the microbiomes of infants that later developed diarrhea were enriched in pathways important for the synthesis of immunomodulatory products such as palmitoleic acid and methylerithrol phosphate (Fig. 5c).

At the 8-month time point, 32 pathways were differentially abundant between infants that remained asymptomatics and those that experienced diarrhea (Fig. 5d and Additional file 2: Table S5). Notably, the fecal microbiomes of monkeys that did not experience diarrhea were enriched in pathways associated with homolactic fermentation and glycolysis. Conversely, the microbiomes of infant macaques that experienced diarrhea were enriched in pathways for aerobic respiration and the breakdown of the SCFA propionate via the methyl citrate cycle most commonly found in Proteobacteria (Fig. 5d, Additional file 2: Table S5).

\section{De novo genome assemblies reveal unique Prevotella and Campylobacter carrier state associate with diarrhea}

Taxonomy assigned using only short reads from shotgun metagenomics libraries can miss organisms that do not have a match in a database, which could be particularly important for studies using samples from NHPs. To address this pitfall, we assembled metagenomic reads of fecal samples into contigs that were subsequently binned into putative genomes. At the 1-month time point, 45 genomes were assembled with a completeness $>80 \%$ and contamination $<2 \%$ from 11 samples (5 diarrhea, 6 asymptomatic, Additional file 2: Table S6). At the 8- 


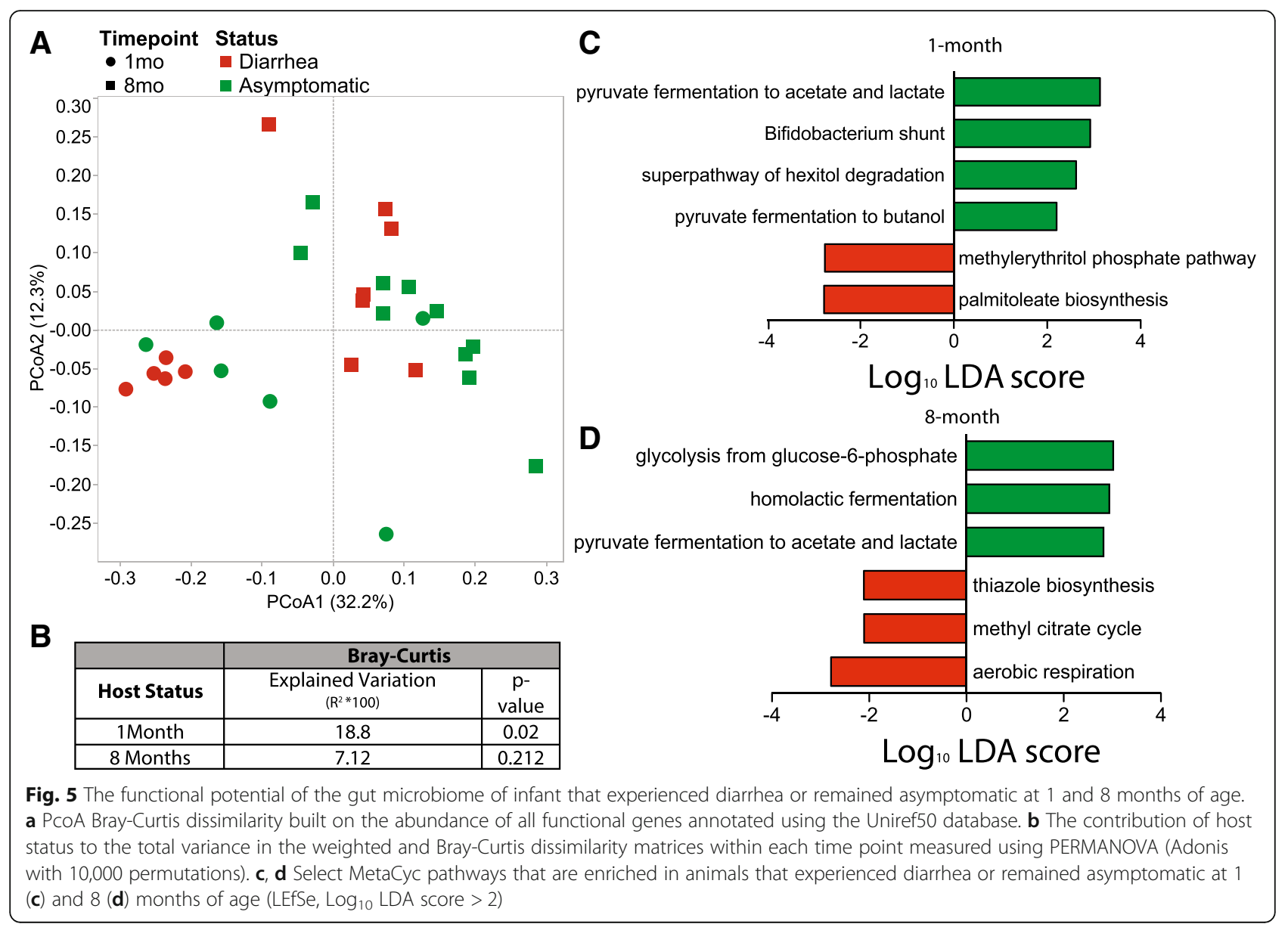

month time point, 50 genomes were assembled with a completeness $>80 \%$ and contamination $<2 \%$ from 17 samples (7 diarrhea, 10 asymptomatic, Additional file 2: Table S6). Six Bifidobacterium genomes assembled from the 1-month samples were closely related to three known Bifidobacterium species, including two that are most closely related to Bifidobacteria kashiwanohense PV20-2 which was assembled from a human infant in Kenya [58] (Additional file 1: Figure S3A). The number of reads that aligned to the six assembled Bifidobacterium genomes significantly decreased between the 1 - and 8-month time point, in line with the 16S rRNA gene amplicon sequencing data and the anticipated decreased in this taxon's abundance after weaning (Additional file 1: Figure S3B).

We also assembled several Prevotella genomes. Interestingly, a clade of five assembled genomes that were only detected in infants that developed diarrhea (Fig. 6a). Three genomes were assembled from 1-month-old samples and two additional genomes were assembled from 8-month-old animals (Fig. 6a and Additional file 2: Table S6). Interestingly, the number of reads that aligned to this clade were significantly higher in samples from the 1-month infants that later experienced diarrhea (Fig. 6b).
Upon annotation, these 5 assembled Prevotella genomes contained 216 unique genes not found in the other assembled Prevotella genomes (Additional file 1: Figure $\mathrm{S} 3 \mathrm{C}$ ). This group of genes included: fliH (type III secretion system), inhA (immune inhibitor A metalloprotease), and nanH (Neuraminidase). When we aligned reads to these 216 genes, we again found that they were significantly more abundant in the samples from 1month infants that would go on to develop diarrhea (Additional file 1: Figure S3D).

Additionally, we identified three Campylobacter spp. genomes from animals that experienced diarrhea from both primate centers. We placed our assembled genomes in phylogeny with related Campylobacter strains from both humans and rhesus macaque based on the alignment of all protein families found in every genome (Fig. 6c). Overall, these genomes were most closely related to Campylobacter fetus and Campylobacter gracilis. However, they were more closely related to each other than any other Campylobacter species already in the PATRIC [59] database. As seen with the $16 \mathrm{~S}$ amplicon sequencing data, the number of reads that aligned to the three assembled Campylobacter genomes were significantly higher in infants that experienced diarrhea at the 


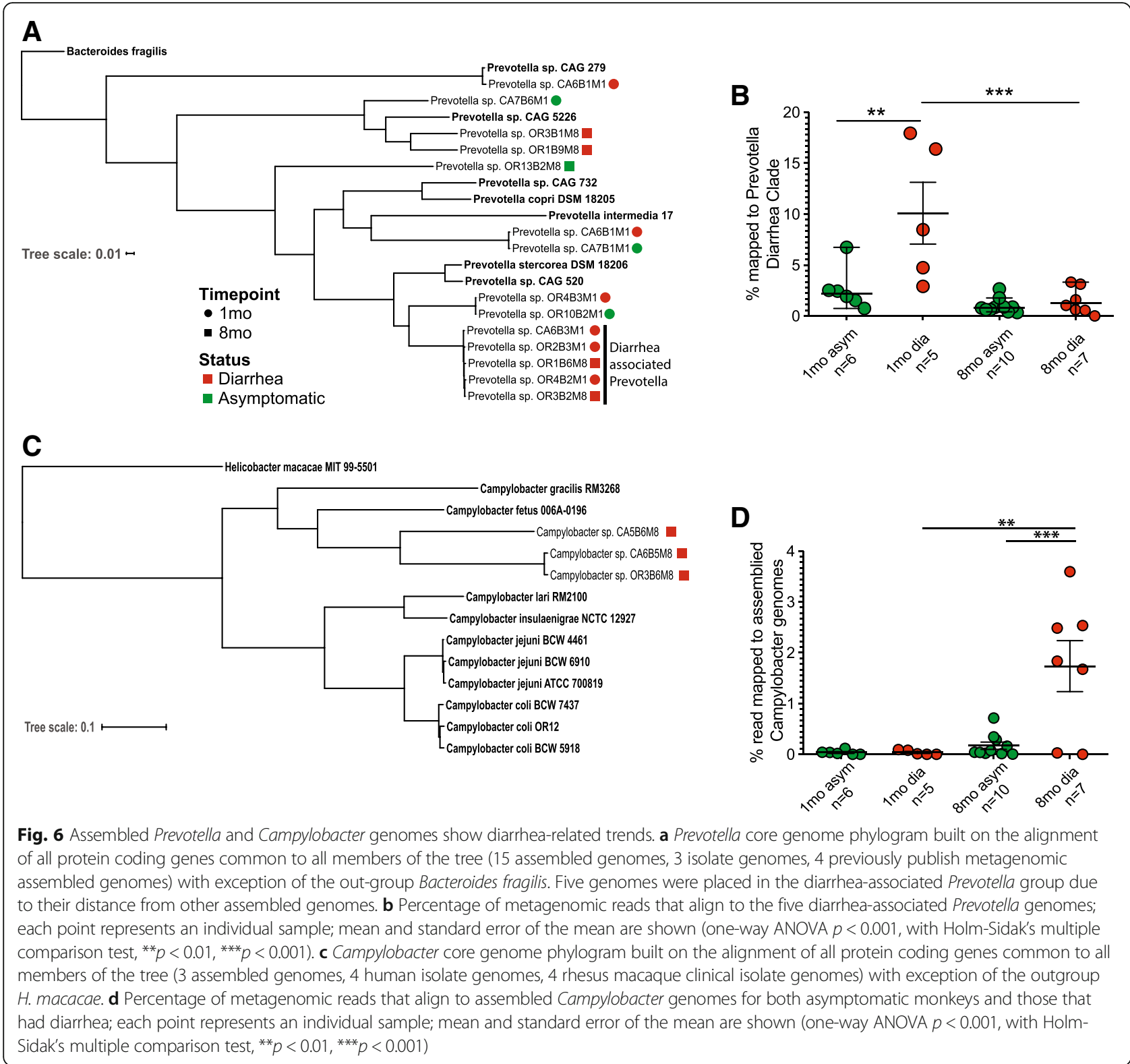

8-month time point when compared to infants that remained asymptomatic and 1-month-old infants from both groups (Fig. 6d).

Additionally, some of the genomes assembled from rectal swabs obtained from animals that experienced diarrhea are closely related to bacteria commonly associated with the human oropharyngeal microbiome. Specifically, three genomes were assigned to the Streptococcus genera and two genomes were identified as Lactobacillus salivarius [60-62] (Additional file 2: Table S6).

\section{Discussion}

In this study, we leveraged the infant rhesus macaque animal model to investigate the role of the microbiome in mediating susceptibility to diarrheal diseases. Here we define the maturation of the infant rhesus macaque gut microbiome through the first 8 months of life, the window during which infant macaques are at the highest risk of developing chronic diarrhea [45, 56]. Additionally, we used shotgun metagenomics to functionally characterize the microbial communities in animals that developed diarrhea versus those that remained asymptomatic before disease onset in order to identify potential biomarkers of susceptibility.

The gut microbiomes of infants and dams in this study show striking similarities to those of humans living in developing countries. Specifically, we report a high abundance of fiber degrading bacteria such as Treponema, Ruminococcace, Fibrobacter, and Lachnospiraceae, which increased with infant age and were found in 
similar abundance in dams as that reported for human adults living in developing countries and huntergatherer societies $[10,12,63]$. Similarities between the gut microbiome of humans and macaques may be due to multiple factors, notably the consumption of plantbased, low-fat diets, which correlates strongly with a community dominated by Prevotella spp. [12, 55], which are sensitive to bile acids produced during the degradation of animal fats [52]. Additionally, poor personal hygiene and sanitation could contribute to increased exposure to enteric pathogens [64].

Our analysis indicates that maturation of the infant rhesus gut microbiome through the first 8 months of life follows similar kinetics as previously reported in humans albeit at a more rapid rate. Specifically, by 8 months of age and after weaning, infant microbiomes were comparable to those of the dams whereas this is achieved at $2-5$ years of age in human infants [10]. This difference is most likely due to the shorter life span of macaques relative to humans ( 1 macaque year is roughly equivalent to 3 human years) as well as earlier weaning and exploration/transition to solid foods. Similar to breastfed human children, infant macaques showed a high abundance of Bifidobacterium, which is quickly diminished after the infant is weaned $[65,66]$. Interestingly, 2 assembled Bifidobacterium genomes were more similar to a genome assembled from an infant in Kenya ( $B$. kashiwanohense) than those typically found in western infants (Bifidobacterium longum, Bifidobacterium adolescentis). In line with this observation, the gut microbial communities detected in infant rhesus macaques were closer to those found in infants living in developing countries than the USA or Italy. Cantenibacterium was not detected after the 3-month time point suggesting that bacteria from this genus may be promoted during breastfeeding. On the other hand, Treponema, Lactobacillus, and Streptococcus increased in abundance with age especially after the 3-month time point when the infants begin exploring solid food.

Despite the shared environment and diet, almost 20\% of the infants in our cohorts experienced and were treated for diarrheal illness by 8 months of age. This suggests that even after controlling for diet and environmental exposure, some individuals are more susceptible to diarrhea due to underlying microbiome, immune status, or genetic background. Analysis of the gut microbiomes of infant that remained asymptomatic throughout the study and those that experienced at least one episode of acute diarrhea revealed potential biomarkers of susceptibility to diarrheal diseases. Although high level bacterial taxonomy information from our $16 \mathrm{~S}$ rRNA gene amplicon sequence data did not show any differential taxa in the pre-diarrhea samples, shotgun metagenomics analysis of the 1-month samples showed significant differences in the overall functional potential, species level taxonomy, and pathway abundance. The microbiota of 1-month-old infant macaques that remained asymptomatic were enriched in Mitsuokela spp. and Lachnospiraceae (5163FAA). Interestingly, Mitsuokela spp. is found in Bangladeshi children but not children from the USA further highlighting the similarities between the gut microbiomes of infant macaques and infants in developing countries [67]. On the other hand, microbiomes of the 1-month-old rhesus infants that later experienced diarrheal disease were enriched for Roseburia intestinalis. This bacterium is believed to dampen inflammation in the gut mucosa by reducing frequency of Th17 CD4 T cells [68]. Its increased abundance in the microbiome of infants that later developed diarrhea could contribute to a reduction in frequency of anti-microbial Th17 $\mathrm{T}$ cells. Interestingly, Lachnospiraceae (5163FAA) was enriched in the microbiomes of infants that did not develop diarrhea at the 1-month time point and in those of infants that developed diarrhea at the 8-month time point. We also found that while the overall microbial composition of gut microbiome became more similar with age, this trend did not hold true for infants that experience diarrhea. These observations could indicate that the microbiomes of infants that experienced diarrhea do not undergo key developmental shifts resulting a more immature gut microbiome. Indeed, some of the microbiomes at the 8-month time point cluster with 1-month microbiomes (Fig. 3a) as also seen in human infants that experienced diarrhea [69].

Through metagenomic genome assembly, we also identified a diarrhea-associated Prevotella clade. These closely related genomes were assembled in samples obtained from both primate centers and time points. Significantly, more reads aligned to these genomes from 1-month-old monkeys that later experienced diarrhea. Prevotella spp. is among the most abundant bacterial taxa in gut microbiota of both humans living in developing countries and rhesus macaques [12, 44, 67]. While typically thought of as a beneficial symbiont aiding the host in the digestion of plant material [70, 71], some strains have been shown to play a role in inflammatory diseases and gut dysbiosis [72, 73]. The 5 genomes assembled from infants that developed diarrhea harbored a number of unique genes. Three of those genes have pathogenic potential. Specifically, bacterial metalloproteases have also been shown to cleave components of the complement system and aid in evasion of the host innate immunity [74]. Type 3 secretion systems are a bacterial mechanism to transfer bacterial proteins into eukaryotic cells and have been proposed as a virulence mechanism for Prevotella associated with periodontal disease [75]. Bacterial Neuraminidases' cleave the sialic acid caps on the host mucin leaving the rest of the 
glycosylated mucin vulnerable to degradation [76, 77], thereby increasing host susceptibility to invasive pathogens such as Campylobacter spp. [78, 79]. Our data suggest that early colonization by some Prevotella spp. may increase susceptibility to diarrheal disease, but additional studies are needed to validate this potential finding.

The microbiomes of infants that remained asymptomatic and those that experienced at least one episode of diarrhea were also functionally distinct at the 1-month time point prior to the development of clinical symptoms. Specifically, microbiomes of infants that remained asymptomatic were enriched in the fermentation pathways of pyruvate to butanol, acetate, and lactate. The degradation of dietary nutrients and resulting production of SCFAs is a key process providing energy to the host, enhancing intestinal barrier function, and communicating with the host immune system [6, 7, 80, 81]. High levels of fermentation, its products, and the resulting anaerobic environment are all thought to indicate a healthy gut microbiota. Microbiomes of infants that developed diarrhea were enriched in the palmitoleate biosynthesis and methylerythritol phosphate pathways, both of which have previously been shown to generate immune modulatory intermediates [82, 83]. Palmitoleic acid has previously been shown to inhibit the production of the pro-inflammatory cytokines IL- $1 \mathrm{a}$ and IL- 6 by human peripheral blood mononuclear cells [82]. A decrease of these cytokines in vivo could hamper the host's ability to respond to infection or could indicate a compensatory mechanism to combat heightened inflammation. Methylerythritol-phosphate plays a role in the activation and differentiation of gamma delta $\mathrm{T}$ cells [83]. Gamma delta $\mathrm{T}$ cells are highly abundant in the gut barrier [84] and differences in their activation could also play a role in diarrhea susceptibility. The products and intermediates of these pathways could potentially be used as biomarkers to determine an individual's susceptibility to diarrheal illness.

We also observed multiple taxonomic and functional differences in the gut microbiome of infant macaques at the 8-month time point in both $16 \mathrm{~S}$ rRNA gene sequencing and shotgun metagenomic data. Differences between the two groups at this time point are likely to reflect shifts in the microbiome due to both disease and antibiotic treatment. Unfortunately, we were unable to disaggregate the role of diarrhea versus treatment since all infants that experienced clinical diarrhea we treated with an antibiotic and received the same probiotic sandwich. One of the main differences is increased abundance of Campylobacter and corresponding decrease of Helicobacter in infants that experienced diarrhea. Although both of these genera fall in the Campylobacterales order, Campylobacter is one of the leading causes of diarrheal illnesses worldwide [85] while Helicobacter has not been associated with diarrheal diseases. Helicobacter and Campylobacter like many gut resident Epsilonproteobacteria are known to colonize the intestinal mucosa $[43,86]$. The low rate of co-occurrence could indicate potential niche competition between a commensal and pathogenic organism; however, the mechanism by which Campylobacter displaces Helicobacter macacae is unclear. Alternatively, these Helicobacter species could be more susceptible to antibiotics than Campylobacter resulting in its depletion. Indeed, a recent study using rhesus macaques reported a large decrease in Helicobacter following vancomycin treatment despite the observation that Helicobacter should not be directly susceptible to vancomycin $[87,88]$.

Assembly of metagenomic reads led to the identification of three novel Campylobacter genomes exclusively in samples obtained from infants that previously had diarrhea. Core genome alignment revealed that our assembled Campylobacter genomes were more similar to each other than previously published genomes of human Campylobacter species. Interestingly, we were unable to assemble $C$. coli or C. lari, which were detected via culture during diarrhea episodes, from the fecal samples analyzed at the 8-month time point. The absence of these two enteropathogens is likely due to two key factors. First, in contrast to culture results, we did not analyze samples collected during acute disease. Second, the conditions required for culturing $C$. coli or C. lari (namely incubation at $42^{\circ} \mathrm{C}$ ) inhibits growth of other Campylobacter species. Our data also suggest that other Campylobacter species could be playing a more critical role in diarrheal diseases in infant macaques than previously appreciated. Indeed, a recent clinical study reported a higher prevalence of other Campylobacter compared to C. coli/jejuni, which were associated with a higher ( 2-fold) burden of severe diarrhea during early childhood [89]. Together with data presented in this manuscript, these data highlight the importance of nonC. coli/jejuni Campylobacter species and the need to clarify their importance in the etiology of clinical disease.

Recent studies have linked altered microbiome composition and assembly to growth stunting showing a reduction in health-associated Succinivibrio and Clostridium in Malawian infants (12-23 months old) [69, 90]. Also, growth-stunted children from Central Africa Republic and Madagascar show small intestine bacterial overgrowth, an increased infiltration of oral microbes throughout the gut, and presence of enteropathogens such as Campylobacter in feces [91]. In line with these studies, we found an increased abundance of oropharyngeal taxa (Lactobacillus, Streptococcus, and Veillonella) in the genomes assembled from 8-month infants that previously experienced 
diarrhea but not those that remained asymptomatic, indicative of compromised compartmentalization.

Functionally, at 8 months, the gut microbiomes of healthy individuals were enriched in pathways for energy catabolism via fermentation indicative of an anaerobic environment that yields energy for the host. In contrast, the gut microbiomes of individuals that experienced diarrhea were enriched in pathways for sulfur metabolism and aerobic respiration indicative of a dysbiotic environment enriched in Proteobacteria able to metabolize sulfur $[92,93]$.

\section{Conclusion}

In summary, this study establishes maturation timeline of the infant rhesus macaque gut microbiome and its association with their adult mothers. Our key findings highlight similarities in development trajectories of the human and macaque infant microbiomes as well as homology of the adult and infant macaque microbiome to that of humans living in poor sanitary conditions and rural communities. Moreover, our data suggest that susceptibility to diarrhea may be impacted by the presence of a microbial community enriched in the potential to produce immunomodulatory products. Diarrhea results in lasting taxonomic and functional shifts in the gut microbiome. These results pave the way to identify potential microbial biomarkers of susceptibility to diarrheal illnesses and suggest novel diagnostic and vaccination strategies.

\section{Methods}

\section{Sample collection and cohort information}

All rhesus macaque studies were overseen and approved by the OHSU/ONPRC and University of California-Davis/ CNPRC Institutional Animal Care and Use Committees' (IACUC) in accordance with the National Institutes of Health guide for the care and use of laboratory animals. Animals were housed in accordance with standards established by the US Federal Animal Welfare Act and The Guide for the Care and Use of Laboratory Animals. All animals were tested annually for simian viruses (Simian Immunodeficiency Virus, Simian Retrovirus 2, Macacine herpesvirus 1, and Simian $\mathrm{T}$ lymphotrophic virus) and received a mammalian old tuberculin test semi-annually. Rectal swabs were collected from 80 infants $(n=40$ from ONPRC, $n=40$ from CNPRC) at 1 and 8 months of age (41 males and 39 females). A subset of 20 of the infants also had swabs collected at 3 and 6 months. At 1 month after birth, rectal swabs were also collected from the dams, who had an average age of 6.5 years (range of 3-19 years of age) at the time of birth (Fig. 1a). Rectal swabs and fecal samples were immediately snap frozen upon collection and stored at $-80^{\circ} \mathrm{C}$ until DNA extraction.
The outdoor-housed NHP at each primate center are naturally exposed to a number of enteric pathogens including Giardia and Cryptosporidium, but routine diagnostic testing of hospitalized diarrhea cases focus mainly on enteric bacterial pathogens such as Campylobacter (C. coli, C. jejuni, at each primate center in addition to C. lari at CNPRC), Shigella (both primate centers), and Yersinia (CNPRC). Shigella colonization was observed in $\geq 20 \%$ of the infants by 1 month of age and $100 \%$ of the infants were colonized with Campylobacter by 6 months of age (manuscript in preparation).

Infant rhesus macaques are exclusively breastfed for the first 3 months of life, after which they begin to explore solid food that the dams are consuming, and are typically completely weaned by $6-7$ months of age. This is only a generalized timeline, and we were unable to collect exact time of weaning for individual infants. Outdoor-housed rhesus macaques are fed twice daily with Lab Diet, Monkey Diet 5038 (Ralston Purina, St Louis, MO, USA). This diet is guaranteed to contain no more than $15 \%$ crude protein, $5 \%$ crude fat, $6 \%$ crude fiber, $9 \%$ ash, and $12 \%$ moisture. This diet is supplemented with seasonal fresh fruit and produce once daily. Municipal water was available ad libitum.

\section{$16 \mathrm{~S}$ rRNA gene library construction and sequencing}

Total DNA was extracted from rectal swabs using the PowerSoil DNA Isolation Kit (MO BIO Laboratories, Carlsbad, CA, USA), and a 30-s bead beating step using a Mini-Beadbeater-16 (BioSpec Products, Bartlesville, OK, USA). This genomic DNA was used as the template to amplify the hypervariable V4 region of the 16S rRNA gene using PCR primers (515F/806R with the reverse primers including a 12-bp barcode) and reactions containing: $50 \mathrm{mM}$ Tris ( $\mathrm{pH} \mathrm{8.3),} 500 \mu \mathrm{g} / \mathrm{ml}$ bovine serum albumin (BSA), $2.5 \mathrm{mM} \mathrm{MgCl} 2,250 \mu \mathrm{M}$ of each deoxynucleotide triphosphate (dNTP), $400 \mathrm{nM}$ of each primer, $5 \mu \mathrm{l}$ of DNA template, and 0.25 units of JumpStart Taq DNA polymerase (Sigma-Aldrich, St Louis, MO, USA). Thermal cycling parameters were $94{ }^{\circ} \mathrm{C}$ for $5 \mathrm{~min} ; 35$ cycles of $94{ }^{\circ} \mathrm{C}$ for $20 \mathrm{~s}, 50^{\circ} \mathrm{C}$ for $20 \mathrm{~s}$, and $72^{\circ} \mathrm{C}$ for $30 \mathrm{~s}$, followed by $72{ }^{\circ} \mathrm{C}$ for $5 \mathrm{~min}$. PCR products were purified using a MinElute 96 UF PCR Purification Kit (Qiagen, Valencia, CA, USA). Libraries were sequenced $(1 \times 300$ bases) using an Illumina MiSeq.

\section{$16 \mathrm{~S}$ rRNA gene sequence processing}

Raw FASTQ 16S rRNA gene amplicon sequences were uploaded and processed using the QIIME2 analysis pipeline [94]. Briefly, sequences were demultiplexed and the quality filtered using DADA2 [95], which filters chimeric sequences and generates sequence variants table equivalent to an operational taxonomic unit (OTU) table at $100 \%$ sequence similarity. Sequence variants were then 
aligned using the MAFFT [96] and a phylogenetic tree was constructed using the FastTree2 program [97]. Taxonomy was assigned to sequence variants using q2feature-classifier [98] against SILVA database (release 119) [99]. To prevent sequencing depth bias, samples were rarified to 13,000 sequences per sample prior to alpha and beta diversity analysis. QIIME 2 was also used to generate the following alpha diversity metrics: richness (as observed taxonomic units), Shannon evenness, and phylogenetic diversity. Beta diversity was estimated in QIIME 2 using weighted and unweighted UniFrac distances [100].

\section{Comparison of infant rhesus macaque gut microbiome to humans}

16S rRNA gene amplicon sequencing data obtained from fecal samples collected from children (6 months old to 6 years old) living in the USA, Malawi, and Venezuela (Amerindians) was obtained from MG-RAST (Accession number: qiime:850) [10]. Additional 16S rRNA gene amplicon sequencing data from fecal samples collected from children (2-6 years old) living in Italy and Burkina Faso were downloaded from the European Nucleotide Archive (Study ID: PRJEB2079) [11]. These samples were then imported to QIIME2 and rarified to 13,000 reads per sample. Taxonomy was assigned using the fulllength SILVA database (release 119) at the 99\% OTU cutoff. Genus level (L6) taxonomy tables were merged, and Bray-Curtis dissimilarity matrices were generated using QIIME2.

\section{Shotgun metagenomics}

Shotgun metagenomic libraries were prepared for a subset of infants that developed diarrhea and a subset of those that remained asymptomatic both at the 1-month time point (prior to the onset of disease, 6 asymptomatic and 5 diarrhea) and at the 8-month time point (after disease resolution, 10 asymptomatic and 7 diarrhea). Libraries were prepared from $50 \mathrm{ng}$ of gDNA using the Illumina Nextera library prep per the manufacturer's recommended protocol and sequenced on an Illumina HiSeq $40002 \times$ 100. Raw demultiplexed reads were quality filtered using Trimmomatic [101], and potential host reads were removed by aligning trimmed reads to the Macaca mulata genome (Mmul 8.0.1) using BowTie2 [102]. After quality filtering and decontamination, an average of 14.25 million reads ( $\min 8.6, \max 20.8$ million reads) per sample were used for downstream analysis. Trimmed and decontaminated reads were then annotated using the HUMAnN2 pipeline using default setting with the UniRef50 database and assigned to MetaCyc pathways. Functional annotations were normalized using copies per million (CPM) reads prior to statistical analysis [103-105]. Species level taxonomy was assigned to quality-controlled short reads using Metaphlan2 [106].

Genome assemblies were generated for each sample individually. Trimmed and decontaminated reads were assembled into contigs using meta-SPAdes with default parameters [107] and binned into putative genomes using MetaBat [108]. Genome completeness/contamination was tested using CheckM [109], and all bins with a completeness $>80 \%$ and contamination $<2 \%$ were annotated using PATRIC [59]. Taxonomy of draft genomes was determined using PATRICs' similar genome finder. Prevotella genomes were annotated and plotted using the Anvi'o pangenomic pipeline [110].

\section{Statistical analysis}

All statistical analyses were conducted using PRISM (V5) and the R package Vegan [111]. QIIME2 was used to calculate alpha-diversity metrics; observed OTUs, Shannon evenness, and beta diversity; and weighted/unweighted UniFrac distances [6]. Bray-Curtis dissimilarity matrices were constructed for both species-level relative abundance, and normalized gene annotations using the vegdist function in the $\mathrm{R}$ package Vegan for shotgun metagenomic data. Principal coordinate analysis (PcoA) was conducted using the $\mathrm{R}$ function cmdscale. PERMANOVAs were performed using the Vegan function ADONIS. Unpaired $t$-test and one-way and two-way ANOVA were implemented using PRISM where noted to generate $p$ values, and utilizing the corresponding post hoc test when the initial ANOVA was significant. The LEfSe algorithm was used to identify differentially abundant taxa and pathways between groups with a logarithmic linear discriminant analysis (LDA) score cutoff of 2 [54].

\section{Additional files}

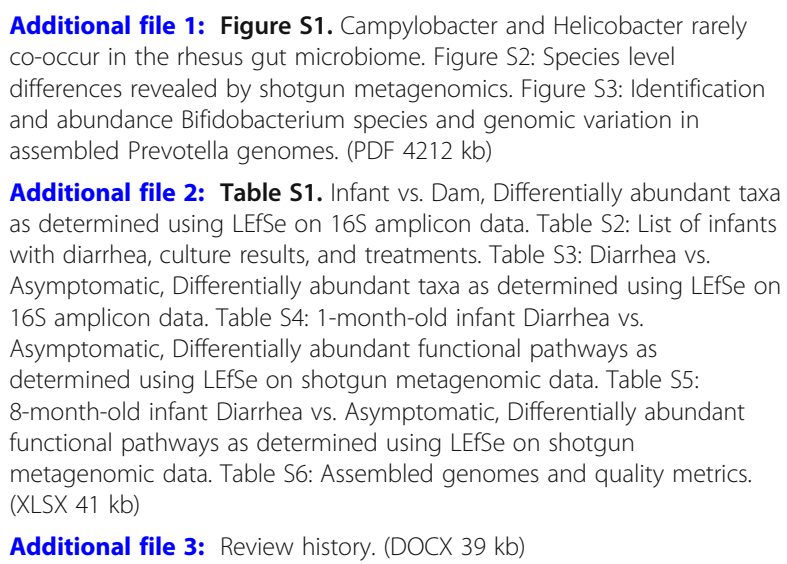

Additional file 2: Table S1. Infant vs. Dam, Differentially abundant taxa as determined using LEfSe on 165 amplicon data. Table S2: List of infants with diarrhea, culture results, and treatments. Table S3: Diarrhea vs. Asymptomatic, Differentially abundant taxa as determined using LEfSe on $16 S$ amplicon data. Table S4: 1-month-old infant Diarrhea vs. Asymptomatic, Differentially abundant functional pathways as determined using LEfSe on shotgun metagenomic data. Table S5: 8-month-old infant Diarrhea vs. Asymptomatic, Differentially abundant functional pathways as determined using LEfSe on shotgun metagenomic data. Table S6: Assembled genomes and quality metrics. (XLSX $41 \mathrm{~kb}$ )

Additional file 3: Review history. (DOCX $39 \mathrm{~kb}$ ) 


\section{Authors' contributions}

IM and MS contributed to the study design. SH, KP, AH, LG, and LG contributed to the sample and clinical data collection and veterinary care. TB and NR contributed to the $16 \mathrm{~S}$ rRNA gene amplicon library generation and sequencing. NR contributed to the shotgun metagenomic sequencing and statistical analysis. KW consulted on shotgun metagenomic data analysis. NR, MS, and IM wrote the manuscript. All authors read and approved the final manuscript.

\section{Funding}

This work was supported by the Bill and Melinda Gates Foundation (OPP1149233) and the US National Institute of Health (NIH) (P51 OD011092). NR was supported by the NIH IMSD fellowship (GM055246).

\section{Availability of data and materials}

The datasets generated and analyzed during the current study are available in the NCBI SRA repository, under the bioproject ID: PRJNA546004 [112]. This data include $16 \mathrm{~S}$ amplicon sequences for 320 samples, unassembled shotgun metagenomic sequences from 28 samples, and 95 metagenomicassembled genomes. For comparison to human samples, $16 \mathrm{~S}$ amplicon data was obtained from Yatsunenko et al. [10] which can be accessed in MG-Rast (Accession number: qiime:850) [113]. Additional human 165 amplicon data was obtained from De Filippo et al. [11] which can be accessed in European Nucleotide Archive (Study ID: PRJEB2079) [114].

\section{Ethics approval and consent to participate}

All rhesus macaque studies were overseen and approved by the OHSU/ ONPRC and University of California-Davis/CNPRC Institutional Animal Care and Use Committees (IACUC: TR01IP00000416 1060).

\section{Competing interests}

The authors declare that they have no competing interests.

\section{Author details}

'Department of Molecular Biology and Biochemistry, University of California Irvine, Irvine, CA, USA. ${ }^{2}$ Division of Neuroscience, Oregon National Primate Research Center, Portland, OR, USA. ${ }^{3}$ Division of Comparative Medicine, Oregon National Primate Research Center, Oregon Health and Science University West Campus, Portland, OR, USA. ${ }^{4}$ California National Primate Research Center, Davis, CA, USA.

Received: 2 January 2019 Accepted: 9 August 2019

Published online: 26 August 2019

\section{References}

1. Turnbaugh PJ, et al. The human microbiome project. Nature. 2007;449:804-10.

2. Costello EK, et al. Bacterial community variation in human body habitats across space and time. Science. 2009;326:1694-7.

3. Ley RE, Peterson DA, Gordon JI. Ecological and evolutionary forces shaping microbial diversity in the human intestine. Cell. 2006;124:837-48.

4. LeBlanc JG, et al. Bacteria as vitamin suppliers to their host: a gut microbiota perspective. Curr Opin Biotechnol. 2013;24:160-8.

5. Magnúsdóttir S, Ravcheev D, de Crécy-Lagard V, Thiele I. Systematic genome assessment of B-vitamin biosynthesis suggests co-operation among gut microbes. Front Genet. 2015;6:148.

6. Peng L, Li ZR, Green RS, Holzman IR, Lin J. Butyrate enhances the intestinal barrier by facilitating tight junction assembly via activation of AMP-activated protein kinase in Caco-2 cell monolayers. J Nutr. 2009;139:1619-25.

7. Morrison DJ, Preston T. Formation of short chain fatty acids by the gut microbiota and their impact on human metabolism. Gut Microbes. 2016;7: 189-200.

8. Galligan JJ. Beneficial actions of microbiota-derived tryptophan metabolites. Neurogastroenterol Motil. 2018;30:e13283.

9. Kennedy PJ, Cryan JF, Dinan TG, Clarke G. Kynurenine pathway metabolism and the microbiota-gut-brain axis. Neuropharmacology. 2017;112:399-412.

10. Yatsunenko T, et al. Human gut microbiome viewed across age and geography. Nature. 2012;486:222-7.

11. De Filippo C, et al. Impact of diet in shaping gut microbiota revealed by a comparative study in children from Europe and rural Africa. Proc Natl Acad Sci U S A. 2010;107:14691-6.
12. Schnorr SL, et al. Gut microbiome of the Hadza hunter-gatherers. Nat Commun. 2014;5:3654.

13. Brussow H. What is health? Microb Biotechnol. 2013;6:341-8.

14. Brussow H. How stable is the human gut microbiota? And why this question matters. Environ Microbiol. 2016;18:2779-83.

15. Packey CD, Sartor RB. Commensal bacteria, traditional and opportunistic pathogens, dysbiosis and bacterial killing in inflammatory bowel diseases. Curr Opin Infect Dis. 2009;22:292-301.

16. Chen SJ, Liu XW, Liu JP, Yang XY, Lu FG. Ulcerative colitis as a polymicrobial infection characterized by sustained broken mucus barrier. World J Gastroenterol. 2014;20:9468-75.

17. Johnson CC, Ownby DR. The infant gut bacterial microbiota and risk of pediatric asthma and allergic diseases. Transl Res. 2016;179:60-70.

18. Martin R, et al. Early-life events, including mode of delivery and type of feeding, siblings and gender, Shape the Developing Gut Microbiota. PLoS One. 2016;11:e0158498.

19. Rutayisire E, Huang K, Liu Y, Tao F. The mode of delivery affects the diversity and colonization pattern of the gut microbiota during the first year of infants' life: a systematic review. BMC Gastroenterol. 2016;16:86.

20. Frese SA, et al. Persistence of supplemented Bifidobacterium longum subsp. infantis EVC001 in breastfed infants. mSphere. 2017;2:e0501-17.

21. Henrick $\mathrm{BM}$, et al. Elevated fecal $\mathrm{pH}$ indicates a profound change in the breastfed infant gut microbiome due to reduction of Bifidobacterium over the past century. mSphere. 2018;3:00041-18.

22. Davis JC, et al. Growth and morbidity of Gambian infants are influenced by maternal milk oligosaccharides and infant gut microbiota. Sci Rep. 2017;7:40466.

23. Charbonneau MR, et al. A microbial perspective of human developmental biology. Nature. 2016;535:48-55.

24. Bailey LC, et al. Association of antibiotics in infancy with early childhood obesity. JAMA Pediatr. 2014;168:1063-9.

25. Murgas Torrazza R, Neu J. The developing intestinal microbiome and its relationship to health and disease in the neonate. J Perinatol. 2011; 31(Suppl 1):S29-34.

26. Stewart CJ, et al. Temporal development of the gut microbiome in early childhood from the TEDDY study. Nature. 2018;562:583-8.

27. Pfefferle PI, Renz H. Microbial exposure and onset of allergic diseases potential prevention strategies? Allergol Int. 2014;63:3-10.

28. Fujimura KE, et al. Neonatal gut microbiota associates with childhood multisensitized atopy and T cell differentiation. Nat Med. 2016;22:1187-91.

29. Branski D, Lerner A, Lebenthal E. Chronic diarrhea and malabsorption. Pediatr Clin N Am. 1996;43:307-31.

30. de Medeiros $P$, et al. Modulation of intestinal immune and barrier functions by vitamin a: implications for current understanding of malnutrition and enteric infections in children. Nutrients. 2018;10:1128.

31. Campbell DI, Elia M, Lunn PG. Growth faltering in rural Gambian infants is associated with impaired small intestinal barrier function, leading to endotoxemia and systemic inflammation. J Nutr. 2003;133:1332-8.

32. John CC, Black MM, Nelson CA. Neurodevelopment: the impact of nutrition and inflammation during early to middle childhood in low-resource settings. Pediatrics. 2017;139:S59-71.

33. Czerkinsky C, Holmgren J. Vaccines against enteric infections for the developing world. Philos Trans R Soc Lond Ser B Biol Sci. 2015;370:20150142.

34. Kim KH, Suh IS, Kim JM, Kim CW, Cho YJ. Etiology of childhood diarrhea in Korea. J Clin Microbiol. 1989:27:1192-6.

35. Germani Y, et al. Two-year study of endemic enteric pathogens associated with acute diarrhea in New Caledonia. J Clin Microbiol. 1994;32:1532-6.

36. Olesen $B$, et al. Etiology of diarrhea in young children in Denmark: a case-control study. J Clin Microbiol. 2005;43:3636-41.

37. Yu J, et al. Etiology of diarrhea among children under the age five in China: results from a five-year surveillance. J Infect. 2015;71:19-27.

38. Harris VC, et al. Significant correlation between the infant gut microbiome and rotavirus vaccine response in rural Ghana. J Infect Dis. 2017;215:34-41.

39. Maldonado J, et al. Human milk probiotic Lactobacillus fermentum CECT5716 reduces the incidence of gastrointestinal and upper respiratory tract infections in infants. J Pediatr Gastroenterol Nutr. 2012;54:55-61.

40. Rasmussen $\mathrm{SH}$, et al. Antibiotic exposure in early life and childhood overweight and obesity: a systematic review and meta-analysis. Diabetes Obes Metab. 2018;20:1508-14.

41. Ville AP, Heyman MB, Medrano R, Wojcicki JM. Early antibiotic exposure and risk of childhood obesity in Latinos. Child Obes. 2017;13:231-5. 
42. Wang $\mathrm{M}$, Monaco MH, Donovan SM. Impact of early gut microbiota on immune and metabolic development and function. Semin Fetal Neonatal Med. 2016:21:380-7.

43. Yasuda $\mathrm{K}$, et al. Biogeography of the intestinal mucosal and lumenal microbiome in the rhesus macaque. Cell Host Microbe. 2015;17:385-91.

44. Clayton JB, et al. Captivity humanizes the primate microbiome. Proc Natl Acad Sci U S A. 2016;113:10376-81.

45. Prongay K, Park B, Murphy SJ. Risk factor analysis may provide clues to diarrhea prevention in outdoor-housed rhesus macaques (Macaca mulatta). Am J Primatol. 2013;75:872-82.

46. Tao N, et al. Evolutionary glycomics: characterization of milk oligosaccharides in primates. J Proteome Res. 2011;10:1548-57.

47. Goto K, et al. Chemical characterization of oligosaccharides in the milk of six species of new and Old World monkeys. Glycoconj J. 2010;27:703-15.

48. Ma J, et al. High-fat maternal diet during pregnancy persistently alters the offspring microbiome in a primate model. Nat Commun. 2014;5:3889.

49. Amaral WZ, et al. Social influences on Prevotella and the gut microbiome of young monkeys. Psychosom Med. 2017;79:888-97.

50. Narayan NR, et al. Persistent effects of early infant diet and associated microbiota on the juvenile immune system. Gut Microbes. 2015;6:284-9.

51. Koenig JE, et al. Succession of microbial consortia in the developing infant gut microbiome. Proc Natl Acad Sci U S A. 2011;108(Suppl 1):4578-85.

52. Hayashi H, Shibata K, Sakamoto M, Tomita S, Benno Y. Prevotella copri sp. nov. and Prevotella stercorea sp. nov., isolated from human faeces. Int J Syst Evol Microbiol. 2007:57:941-6.

53. Gueimonde M, Laitinen K, Salminen S, Isolauri E. Breast milk: a source of bifidobacteria for infant gut development and maturation? Neonatology. 2007:92:64-6.

54. Segata N, et al. Metagenomic biomarker discovery and explanation. Genome Biol. 2011;12:R60.

55. De Filippo C, et al. Diet, environments, and gut microbiota. A preliminary investigation in children living in rural and urban Burkina Faso and Italy. Front Microbiol. 2017;8:1979.

56. Haertel AJ, Prongay K, Gao L, Gottlieb DH, Park B. Standard growth and diarrhea-associated growth faltering in captive infant rhesus macaques (Macaca mulatta). Am J Primatol. 2018;80:e22923.

57. Gough EK, et al. Linear growth faltering in infants is associated with Acidaminococcus sp. and community-level changes in the gut microbiota. Microbiome. 2015;3:24.

58. Vazquez-Gutierrez $P$, et al. Complete and assembled genome sequence of Bifidobacterium kashiwanohense PV20-2, Isolated from the Feces of an Anemic Kenyan Infant. Genome Announc. 2015;3:e01467-14.

59. Wattam AR, et al. Assembly, annotation, and comparative genomics in PATRIC, the all bacterial bioinformatics resource center. Methods Mol Biol. 2018;1704:79-101.

60. Costalonga M, Herzberg MC. The oral microbiome and the immunobiology of periodontal disease and caries. Immunol Lett. 2014;162:22-38.

61. Carlsson J, Grahnén H, Jonsson G. Lactobacilli and streptococci in the mouth of children. Caries Res. 1975;9:333-9.

62. Caufield PW, Schön CN, Saraithong P, Li Y, Argimón S. Oral lactobacilli and dental caries: a model for niche adaptation in humans. J Dent Res. 2015;94: 110S-8S.

63. Ayeni FA, et al. Infant and adult gut microbiome and metabolome in rural Bassa and urban settlers from Nigeria. Cell Rep. 2018;23:3056-67.

64. Guerrant RL, DeBoer MD, Moore SR, Scharf RJ, Lima AA. The impoverished gut--a triple burden of diarrhoea, stunting and chronic disease. Nat Rev Gastroenterol Hepatol. 2013;10:220-9.

65. Lewis ZT, Mills DA. Differential establishment of Bifidobacteria in the breastfed infant gut. Nestle Nutr Inst Workshop Ser. 2017;88:149-59.

66. Roger LC, Costabile A, Holland DT, Hoyles L, McCartney AL. Examination of faecal Bifidobacterium populations in breast- and formula-fed infants during the first 18 months of life. Microbiology. 2010;156:3329-41.

67. Lin A, et al. Distinct distal gut microbiome diversity and composition in healthy children from Bangladesh and the United States. PLoS One. 2013;8: e53838.

68. Zhu C, et al. Roseburia intestinalis inhibits interleukin17 excretion and promotes regulatory T cells differentiation in colitis. Mol Med Rep. 2018;17:7567-74.

69. Subramanian $\mathrm{S}$, et al. Persistent gut microbiota immaturity in malnourished Bangladeshi children. Nature. 2014;510:417-21.

70. David LA, et al. Diet rapidly and reproducibly alters the human gut microbiome. Nature. 2014;505:559-63.
71. El Kaoutari A, Armougom F, Gordon Jl, Raoult D, Henrissat B. The abundance and variety of carbohydrate-active enzymes in the human gut microbiota. Nat Rev Microbiol. 2013;11:497-504.

72. Larsen JM. The immune response to Prevotella bacteria in chronic inflammatory disease. Immunology. 2017;151:363-74.

73. Zhong $\mathrm{D}$, Wu $\mathrm{C}$, Zeng $\mathrm{X}$, Wang $\mathrm{Q}$. The role of gut microbiota in the pathogenesis of rheumatic diseases. Clin Rheumatol. 2018;37:25-34.

74. Laarman AJ, et al. Staphylococcus aureus metalloprotease aureolysin cleaves complement C3 to mediate immune evasion. J Immunol. 2011;186:6445-53.

75. Zhang $Y$, et al. Population-genomic insights into variation in Prevotella intermedia and Prevotella nigrescens isolates and its association with periodontal disease. Front Cell Infect Microbiol. 2017;7:409.

76. Soong $\mathrm{G}$, et al. Bacterial neuraminidase facilitates mucosal infection by participating in biofilm production. J Clin Invest. 2006;116:2297-305.

77. Almagro-Moreno S, Boyd EF. Insights into the evolution of sialic acid catabolism among bacteria. BMC Evol Biol. 2009;9:118.

78. Alemka A, Corcionivoschi N, Bourke B. Defense and adaptation: the complex inter-relationship between campylobacter jejuni and mucus. Front Cell Infect Microbiol. 2012;2:15.

79. Desai MS, et al. A dietary fiber-deprived gut microbiota degrades the colonic mucus barrier and enhances pathogen susceptibility. Cell. 2016;167:1339-1353 e1321.

80. den Besten $\mathrm{G}$, et al. The role of short-chain fatty acids in the interplay between diet, gut microbiota, and host energy metabolism. J Lipid Res. 2013;54:2325-40.

81. D'Souza WN, et al. Differing roles for short chain fatty acids and GPR43 agonism in the regulation of intestinal barrier function and immune responses. PLoS One. 2017;12:e0180190.

82. Schirmer $M$, et al. Linking the human gut microbiome to inflammatory cytokine production capacity. Cell. 2016;167:1897.

83. Feurle J, et al. Escherichia coli produces phosphoantigens activating human gamma delta T cells. J Biol Chem. 2002;277:148-54.

84. Hayday AC. Gammadelta T cells and the lymphoid stress-surveillance response. Immunity. 2009;31:184-96.

85. Kaakoush NO, Castano-Rodriguez N, Mitchell HM, Man SM. Global epidemiology of campylobacter infection. Clin Microbiol Rev. 2015;28:687-720.

86. Romano-Keeler J, et al. Early life establishment of site-specific microbial communities in the gut. Gut Microbes. 2014;5:192-201.

87. Ortiz AM, et al. Experimental microbial dysbiosis does not promote disease progression in SIV-infected macaques. Nat Med. 2018;24:1313-6.

88. Laing ST, et al. Idiopathic colitis in rhesus macaques is associated with dysbiosis, abundant enterochromaffin cells and altered T-cell cytokine expression. Vet Pathol. 2018;55:741-52

89. François $\mathrm{R}$, et al. The other Campylobacters: not innocent bystanders in endemic diarrhea and dysentery in children in low-income settings. PLoS Negl Trop Dis. 2018;12:e0006200.

90. Ordiz Ml, et al. Environmental enteric dysfunction and the fecal microbiota in Malawian children. Am J Trop Med Hyg. 2017;96:473-6.

91. Vonaesch P, et al. Stunted childhood growth is associated with decompartmentalization of the gastrointestinal tract and overgrowth of oropharyngeal taxa. Proc Natl Acad Sci U S A. 2018;115(36):E8489-98.

92. Litvak Y, Byndloss MX, Tsolis RM, Bäumler AJ. Dysbiotic Proteobacteria expansion: a microbial signature of epithelial dysfunction. Curr Opin Microbiol. 2017;39:1-6.

93. Shin NR, Whon TW, Bae JW. Proteobacteria: microbial signature of dysbiosis in gut microbiota. Trends Biotechnol. 2015;33:496-503.

94. R. J. Bolyen E, Dillon MR, Bokulich NA, Abnet C, Al-Ghalith GA, Alexander H, Alm EJ, Arumugam M, Asnicar F, Bai Y, Bisanz JE, Bittinger K, Brejnrod A, Brislawn CJ, Brown CT, Callahan BJ, Caraballo-Rodríguez AM, Chase J, Cope E, Da Silva R, Dorrestein PC, Douglas GM, Durall DM, Duvallet C, Edwardson CF, Ernst M, Estaki M, Fouquier J, Gauglitz JM, Gibson DL, Gonzalez A, Gorlick K, Guo J, Hillmann B, Holmes S, Holste H, Huttenhower C, Huttley G, Janssen S, Jarmusch AK, Jiang L, Kaehler B, Kang KB, Keefe CR, Keim P, Kelley ST, Knights D, Koester I, Kosciolek T, Kreps J, Langille MG, Lee J, Ley R, Liu Y, Loftfield E, Lozupone C, Maher M, Marotz C, Martin BD, McDonald D, Mclver LJ, Melnik AV, Metcalf JL, Morgan SC, Morton J, Naimey AT, Navas-Molina JA, Nothias LF, Orchanian SB, Pearson T, Peoples SL, Petras D, Preuss ML, Pruesse E, Rasmussen LB, Rivers A, Robeson, II MS, Rosenthal P, Segata N, Shaffer M, Shiffer A, Sinha R, Song SJ, Spear JR, Swafford AD, Thompson LR, Torres PJ, Trinh P, Tripathi A, Turnbaugh PJ, UI-Hasan S, van der Hooft JJ, Vargas F, Vázquez-Baeza $Y$, Vogtmann $E$, von Hippel $M$, Walters W, Wan $Y$, Wang M, Warren J, Weber KC, Williamson CH, Willis AD, Xu ZZ, Zaneveld JR, 
Zhang Y, Zhu Q, Knight R, Caporaso JG, QIIME 2: Reproducible, interactive, scalable, and extensible microbiome data science. PeerJ. 2018;6:e27295v2.

95. Callahan BJ, et al. DADA2: high-resolution sample inference from Illumina amplicon data. Nat Methods. 2016:13:581-3.

96. Katoh K, Standley DM. MAFFT multiple sequence alignment software version 7: improvements in performance and usability. Mol Biol Evol. 2013;30:772-80.

97. Price MN, Dehal PS, Arkin AP. FastTree 2--approximately maximum-likelihood trees for large alignments. PLoS One. 2010;5:e9490.

98. Bokulich NA, et al. Optimizing taxonomic classification of marker-gene amplicon sequences with QIIME 2's q2-feature-classifier plugin. Microbiome. 2018;6:90.

99. Quast C, et al. The SILVA ribosomal RNA gene database project: improved data processing and web-based tools. Nucleic Acids Res. 2013;41:D590-6.

100. Lozupone C, Lladser ME, Knights D, Stombaugh J, Knight R. UniFrac: an effective distance metric for microbial community comparison. ISME J. 2011; 5:169-72.

101. Bolger AM, Lohse M, Usadel B. Trimmomatic: a flexible trimmer for Illumina sequence data. Bioinformatics. 2014;30:2114-20.

102. Langmead B, Salzberg SL. Fast gapped-read alignment with bowtie 2. Nat Methods. 2012;9:357-9.

103. Suzek BE, Wang Y, Huang H, McGarvey PB, Wu CH. UniRef clusters: a comprehensive and scalable alternative for improving sequence similarity searches. Bioinformatics. 2015;31:926-32.

104. Abubucker $\mathrm{S}$, et al. Metabolic reconstruction for metagenomic data and its application to the human microbiome. PLoS Comput Biol. 2012;8:e1002358.

105. Karp PD, Riley M, Paley SM, Pellegrini-Toole A. The MetaCyc Database. Nucleic Acids Res. 2002;30:59-61.

106. Segata $\mathrm{N}$, et al. Metagenomic microbial community profiling using unique clade-specific marker genes. Nat Methods. 2012;9:811-4.

107. Bankevich A, et al. SPAdes: a new genome assembly algorithm and its applications to single-cell sequencing. J Comput Biol. 2012;19:455-77.

108. Kang DD, Froula J, Egan R, Wang Z. MetaBAT, an efficient tool for accurately reconstructing single genomes from complex microbial communities. PeerJ. 2015;3:e1165.

109. Parks DH, Imelfort M, Skennerton CT, Hugenholtz P, Tyson GW. CheckM: assessing the quality of microbial genomes recovered from isolates, single cells, and metagenomes. Genome Res. 2015;25:1043-55.

110. Delmont TO, Eren AM. Linking pangenomes and metagenomes: the Prochlorococcus metapangenome. PeerJ. 2018;6:e4320.

111. P. Dixon, VEGAN, a package of $R$ functions for community ecology. Journal of Vegetation Science. 2003;14: 927-930.

112. N. Rhoades, I. Messaoudi, Maturation of the infant rhesus macaque gut microbiome and its role in the development of diarrheal disease. 165 amplicon, Shotgun metagenomics, Assembled Genomes, NCBI:BioProject: PRJNA546004. http://www.ncbi.nlm.nih.gov/bioproject/PRJNA546004 . 2019; Accessed in June 2019

113. T. Yatsunenko, Human gut microbiome viewed across age and geography. $16 S$ amplicon. MG-RAST:Study:mgp401. https://www.mg-rast.org/linkin. cgi?project=mgp401 . 2012; Accessed in June 2019.

114. C. De Filippo, The dominant role of diet on gut microbiota: comparative study in children from Europe and rural Africa. $16 \mathrm{~S}$ amplicon. European Nucleotide Archive:Study ID: PRJEB2079. https://www.ebi.ac.uk/ena/data/ view/PRJEB2079 . 2010; Accessed in June 2019.

\section{Publisher's Note}

Springer Nature remains neutral with regard to jurisdictional claims in published maps and institutional affiliations.

Ready to submit your research? Choose BMC and benefit from:

- fast, convenient online submission

- thorough peer review by experienced researchers in your field

- rapid publication on acceptance

- support for research data, including large and complex data types

- gold Open Access which fosters wider collaboration and increased citations

- maximum visibility for your research: over $100 \mathrm{M}$ website views per year

At BMC, research is always in progress.

Learn more biomedcentral.com/submissions 